CLINICAL STUDY

\title{
Cardiac manifestations of GH deficiency after treatment for acromegaly: a comparison to patients with biochemical remission and controls
}

\author{
Agatha A van der Klaauw ${ }^{1}$, Jeroen J Bax ${ }^{2}$, Gabe B Bleeker ${ }^{2}$, Eduard R Holman ${ }^{2}$, V Delgado ${ }^{2}$, Johannes W A Smit ${ }^{1}$, \\ Johannes A Romijn ${ }^{1}$ and Alberto M Pereira ${ }^{1}$ \\ Departments of ${ }^{1}$ Endocrinology and Metabolic Diseases, C4-R and ${ }^{2}$ Cardiology, Leiden University Medical Center, PO Box 9600,2300 RC Leiden, \\ The Netherlands \\ (Correspondence should be addressed to A A van der Klaauw; Email: a.a.van_der_klaauw@lumc.nl)
}

\begin{abstract}
Objective: Both GH excess and GH deficiency (GHD) lead to specific cardiac pathology. The aim of this study was to evaluate cardiac morphology and function in patients with GHD after treatment for acromegaly. Design: Cross-sectional study.

Patients and methods: Cardiac parameters were studied by conventional two-dimensional echocardiography and tissue Doppler imaging in 53 patients with acromegaly (16 patients with GHD, 20 patients with biochemical remission, and 17 patients with active disease). Patients with GHD were also compared with age- and gender-matched controls.

Results: Left ventricular (LV) dimensions, wall thickness, and mass did not differ between the three groups, or between the patients with GHD and healthy controls. Systolic function, assessed by LV ejection fraction, tended to be lower in patients with GHD compared with patients with biochemical remission $(65.9 \pm 7.3 \%$ vs $72.4 \pm 8.5 \%, P=0.070)$, but was higher when compared with active acromegaly $(58.8 \pm 9.3 \%$, $P=0.047)$. No differences were found with healthy controls. Diastolic function, measured with early diastolic velocity $\left(E^{\prime}\right)$, was lower in patients with $\mathrm{GHD}$ when compared with both patients with biochemical remission $(6.0 \pm 2.1 \mathrm{~cm} / \mathrm{s}$ vs $8.3 \pm 1.5 \mathrm{~cm} / \mathrm{s}, P=0.005)$ and healthy controls $(8.1 \pm 1.9 \mathrm{~cm} / \mathrm{s}, P=0.006)$. Conclusion: $\mathrm{GHD}$ after acromegaly results in a specific decrease in diastolic function compared with patients with biochemical remission of acromegaly and healthy controls. In addition, systolic function tends to be decreased in patients with GHD compared with patients with biochemical remission, but was higher than that in patients with active acromegaly.
\end{abstract}

European Journal of Endocrinology 159 705-712

\section{Introduction}

Acromegaly is associated with increased cardiovascular morbidity and mortality (1). Active disease leads to specific cardiac pathology, which involves the myocardium, the conduction system, and the valves (1). As a consequence, clinical manifestations include biventricular concentric hypertrophy, left ventricular (LV) systolic and diastolic dysfunction, arrhythmias, and valvular regurgitation.

Treatment of GH excess can normalize mortality (2) and reverse heart failure and myocardial hypertrophy (3, 4). However, surgical treatment of GH excess followed by radiotherapy can result in GH deficiency (GHD) (5) and GHD per se is also associated with cardiomyopathy. Cardiac manifestations of GHD include a decrease in LV mass and LV ejection fraction (LVEF) (6-12), which is correlated to the severity of GHD (8). Additionally, impairment in diastolic function has also been observed in patients with GHD (13). Therefore, GHD after successful treatment of acromegaly may be another part of the spectrum of cardiac manifestations of acromegaly.

However, it is presently unknown if, and to what extent, the heart can adapt to prolonged, sequential exposure to GH excess and GHD. Therefore, the aim of this study was to make a detailed assessment of cardiac function and morphology in patients with GHD after treatment for acromegaly, and to compare these data with those obtained in patients with biochemical remission of acromegaly and patients with active acromegaly.

\section{Patients and methods}

\section{Patients}

We studied 16 patients with GHD after successful treatment of acromegaly (eight men) with a mean age of $56 \pm 12$ years. We compared the parameters of these patients with patients with active acromegaly and patients 
with biochemical remission of acromegaly, which were previously reported in studies that assessed the prevalence of valvular regurgitation (14) and diastolic dysfunction in acromegaly (15). Since there could be residual cardiac manifestations of previous $\mathrm{GH}$ excess in patients with biochemical remission from acromegaly, we also compared the patients with GHD after successful treatment of acromegaly with healthy controls.

Inclusion criteria were:

i. GHD after treatment for acromegaly $(n=16)$ : defined as a subnormal $\mathrm{GH}$ response to the insulin tolerance test (short-acting insulin 0.05-0.1 U/kg body weight s.c., blood samples drawn at $0,20,30,45,60$, and $90 \mathrm{~min}$; nadir glucose levels were all below $2.2 \mathrm{mmol} / \mathrm{l}$ ). The increase in $\mathrm{GH}$ concentrations was considered insufficient, if peak $\mathrm{GH}$ response was below $3 \mu \mathrm{g} / \mathrm{l}(5,16)$. Previous treatment of these patients consisted of surgery and radiotherapy $(n=15)$, or surgery only $(n=1)$. Radiotherapy was applied 17.9 years (range 4-29 years) prior to inclusion in the present study. Patients were studied just before the start of rhGH replacement.

ii. Active acromegaly $(n=17)$ : defined as mean fasting $\mathrm{GH}$ concentrations (measured every $30 \mathrm{~min}$ for $3 \mathrm{~h}$ ) $>2.5 \mu \mathrm{g} / \mathrm{l}$, and elevated age- and gender-adjusted IGF1 concentrations. These patients consisted of two groups: a) untreated acromegaly $(n=8)-$ no treatment to reduce GH excess had yet been instituted; b) uncontrolled acromegaly $(n=9)$ - elevated mean plasma GH and IGF1 concentrations despite maximal dosages of Depot octreotide acetate (30 mg i.m. every 3 weeks).

iii. Biochemical remission of acromegaly $(n=20)$ : defined as mean fasting $\mathrm{GH}$ concentrations (measured for $3 \mathrm{~h}$ with an interval of $30 \mathrm{~min})<2.5 \mu \mathrm{g} / \mathrm{l}$, and normal age- and gender-adjusted IGF1 concentrations. These patients consisted of two groups: a) well-controlled acromegaly $(n=14)$ - biochemical control of $\mathrm{GH}$ excess during treatment with somatostatin analogs; b) cured acromegaly $(n=6)$ - no GH excess after surgery only $(n=5)$ or primary radiotherapy $(n=1)$.

iv. Healthy controls $(n=16)$ : the patients with $\mathrm{GHD}$ after acromegaly were compared with 16 healthy age-, body surface area-, and sex-matched controls. The controls were selected from a database with patients referred to the department of Cardiology, based on age, sex, and body surface area. Controls were excluded when referred for echocardiographic evaluation of known valvular heart disease, murmur, congestive heart failure, and cardiac transplantation. Other exclusion criteria were myocardial infarction, thyreotoxicosis, rheumatic fever, endocarditis, connective tissue disease, carcinoid syndrome, or use of anorectic drugs. We and others have previously demonstrated that recruitment of controls from a large database can also be used as representative controls $(14,17)$.
None of the patients had hemodynamic instability, previous myocardial infarction, thyreotoxicosis, rheumatic fever, endocarditis, or connective tissue disease. The medical ethics committee of the Leiden University Medical Center approved the study, and written informed consent was obtained from all subjects.

\section{Echocardiography}

Echocardiography was performed while the patients were in the left lateral decubitus position using a commercially available system (Vingmed Vivid-7, General Electric-Vingmed, Milwaukee, WI, USA). Standard parasternal (long and short axis) and apical views (2- and 4-, and long axis) were obtained.

M-mode images were obtained from the parasternal long-axis views for quantitative assessment of $\mathrm{LV}$ dimensions (interventricular septum thickness (IVST), posterior wall thickness (PWT), LV end-diastolic diameter (LVEDD), LV end-systolic diameter (LVESD), fractional shortening (FS), and LVEF) (18).

The following parameters of diastolic function were obtained: diastolic transmitral peak velocities ( $E$ and $A$ wave) and the $E / A$ ratio. Quantitative diastolic data were derived from tissue Doppler imaging (TDI). For TDI analysis, the digital cine-loops were analyzed using commercial software (Echopac 6.1; General ElectricVingmed). The sample volume $\left(4 \mathrm{~mm}^{2}\right)$ was placed in the LV basal portion of the septum (using the fourchamber views). The following parameters (mean values calculated from three consecutive heartbeats) were derived: early diastolic velocity $\left(E^{\prime}\right)$, late diastolic velocity $\left(A^{\prime}\right)$, and the $E^{\prime} / A^{\prime}$ ratio.

The severity of valvular regurgitation was assessed by two independent expert readers blinded to the clinical data on a qualitative scale of trace, mild, moderate, or severe, using previously described methods (19, 20).

LV mass (LVM) was calculated by the cube formula, and using the correction formula proposed by Devereux et al. (21): $0.8 \times\left(1.04 \quad\left(\left(\mathrm{LVEDD}+\mathrm{PWT}+\mathrm{IVST}^{3}-\right.\right.\right.$ $\left.\left.\mathrm{LVEDD}^{3}\right)\right)+0.6$. LVM indexation (LVMi) was corrected for body height (22). LV hypertrophy (LVH) was defined as LVMi exceeding $49.2 \mathrm{~g} / \mathrm{m}^{2.7}$ for men and $46.7 \mathrm{~g} / \mathrm{m}^{2.7}$ for women (22).

\section{Assays}

GH concentrations were quantitated using a sensitive time-resolved immunofluorescent assay (Wallac Oy, Turku, Finland), specific for $22 \mathrm{kDa} G \mathrm{GH}$ protein. The detection limit was $0.012 \mu \mathrm{g} / \mathrm{l}$. Inter-assay coefficients of variation were $8.4-1.6 \%$ in the $\mathrm{GH}$ range $0.1-18 \mu \mathrm{g} / \mathrm{l}$ $(1 \mu \mathrm{g} / \mathrm{l}=2.6 \mathrm{mU} / \mathrm{l})$. Total serum IGF1 concentration was determined by RIA after extraction and purification on ODS-silica columns (Incstar Corp., Stillwater, MN, USA). The intra- and inter-assay coefficients of variation were less than $11 \%$. The detection limit was 
$1.5 \mathrm{nmol} / \mathrm{l}$. Age- and gender-adjusted IGF1 data were determined in the same laboratory. IGF1 was expressed as a SDS for age- and gender-related normal levels, using $\lambda-\mu-\sigma$ smoothed reference curves based on measurements in 906 healthy individuals $(23,24)$.

A Hitachi 800 autoanalyzer (Roche) was used to quantify serum concentrations of glucose, total cholesterol (TC), and triglycerides (TG). High density lipoprotein (HDL) cholesterol was measured with a homogenous enzymatic assay (Hitachi 911, Roche). Low density lipoprotein (LDL) concentrations were calculated using the Friedewald formula. Unfortunately, lipid concentrations at the time of echocardiography were only available in 9 out of 17 patients with active acromegaly.

\section{Statistical analysis}

Statistical analysis was performed using SPSS for Windows, version 14.0 (SPSS Inc. Chicago, IL, USA). Results are expressed as the mean \pm s.D., unless specified otherwise. ANOVA analysis with Tukey HSD correction for multiple comparisons was used to compare patients with GHD after acromegaly with patients with biochemical remission and patients with active acromegaly. We checked all comparisons after log transformation of the variables. Results were also checked after adjustment for age by ANCOVA. Independent samples $t$-tests and $\chi^{2}$ tests were used to compare patients with GHD after acromegaly and healthy controls. In addition, regression analysis was performed with systolic and diastolic function as dependent variables and age, body mass index (BMI), IGF1 SDS, hypertension, and LVH as independent variables to identify predictors of cardiac function in patients with acromegaly. A $P$ value $<0.05$ was considered to represent a significant difference.

\section{Results}

\section{Clinical characteristics}

Age and gender were not different between the three patient groups (Table 1). GH concentrations and IGF1 SDS were lower in the patients with GHD after acromegaly compared with patients with active acromegaly $(P<0.001$ and $P<0.001$, respectively). GH and IGF1 concentrations did not differ between patients with GHD after acromegaly and patients with biochemical remission $(P=0.839$ and $P=0.195$, respectively). In patients with GHD after acromegaly, the interval between diagnosis of GHD and this study was $3.2 \pm 0.9$ years. In addition, the interval between biochemical remission and the insulin tolerance test (ITT) was $12.8 \pm 7.0$ years. The interval between diagnosis and remission was $4.1 \pm 5.1$ years.

In patients with biochemical remission, the interval between disease remission and this study was $6.4 \pm 4.9$ years, and that between diagnosis and remission $3.8 \pm$ 4.5 years.

In patients with active acromegaly, the estimated disease duration was $14.5 \pm 10.8$ years. The number of patients with hypertension in the active disease group was $(n=7,41 \%)$, in the biochemical remission group $(n=3,15 \%)$ and $\mathrm{GHD}(n=6,38 \%$, overall $P$ value $0.168)$. None of the patients with GHD after acromegaly compared with two patients with active acromegaly (12\%) and two with biochemical remission (10\%) suffered from diabetes mellitus (overall $P$ value $=$ $0.384)$. Two of the patients with GHD after acromegaly (13\%) compared with one with active acromegaly (5\%) and one with biochemical remission (5\%) used lipid lowering drugs (overall $P$ value $=0.665$ ).

ACTH deficiency was present and substituted in nine patients with GHD after acromegaly (56\%), three patients with biochemical remission (15\%), and two

Table 1 Clinical characteristics of patients with GH deficiency after acromegaly compared with patients with biochemical remission of acromegaly and patients with active acromegaly and healthy controls.

\begin{tabular}{lllll}
\hline & $\begin{array}{l}\text { GHD after acromegaly } \\
(n=16)\end{array}$ & $\begin{array}{l}\text { Biochemical remission of } \\
\text { acromegaly }(n=20)\end{array}$ & $\begin{array}{l}\text { Active acromegaly } \\
(n=17)\end{array}$ & $\begin{array}{l}\text { Healthy controls } \\
(n=16)^{\mathrm{a}}\end{array}$ \\
\hline Age (years) & $56 \pm 12$ & $57 \pm 13$ & $54 \pm 16$ & $56 \pm 6$ \\
Gender (male/female (\%)) & $50 / 50$ & $50 / 50$ & $53 / 47$ & $50 / 50$ \\
BMI (kg/m ${ }^{2}$ ) & $30.2 \pm 4.5^{*}$ & $26.8 \pm 4.1$ & $28.7 \pm 3.8$ & \\
GH (mU/l) & $0.6 \pm 0.4^{\dagger}$ & $2.4 \pm 0.5$ & $9.1 \pm 5.1$ \\
IGF1 (SDS) & $-0.7 \pm 1.7^{\dagger}$ & $1.1 \pm 1.7$ & $5.3 \pm 1.1$ \\
Total cholesterol (mmol/l) & $6.1 \pm 1.0$ & $5.3 \pm 1.1$ & $3.7 \pm 0.8$ \\
LDL cholesterol (mmol/l) & $4.2 \pm 0.9$ & $3.7 \pm 1.0$ & $1.6 \pm 0.4$ \\
HDL cholesterol (mmol/l) & $1.4 \pm 0.5$ & $1.8 \pm 1.0$ & $1.3 \pm 0.4$ \\
Triglycerides (mmol/l) & $2.1 \pm 1.3$ & $1.3 \pm 0.5$ & 29 & \\
Surgery (\%) & 100 & 75 & 12 & \\
Radiotherapy (\%) & 94 & 15 & 53 & \\
Somatostatin analogs (\%) & NA & 70 & 47 \\
No treatment yet (\%) & NA & NA & \\
\hline
\end{tabular}

${ }^{\star} P<0.05$ compared with patients with biochemical remission of acromegaly in an ANOVA with Tukey HSD post hoc comparison. ${ }^{\dagger} P<0.05$ compared with patients with active acromegaly in an ANOVA with Tukey HSD post hoc comparison.

${ }^{a}$ Healthy controls were age-, gender and BSA matched to the patients with GHD after acromegaly. 
patients with active acromegaly (5\%). TSH deficiency was present and substituted in five patients with GHD after acromegaly (31\%), one patient with biochemical remission $(5 \%)$, and one patient with active acromegaly (6\%). Three male patients and two female patients with GHD after acromegaly were treated with testosterone and estrogen substitution respectively. Three male patients with biochemical remission of acromegaly and three male patients with active acromegaly were treated with testosterone substitution. None of the female patients with biochemical remission or active acromegaly needed estrogen substitution.

\section{GHD after acromegaly compared with biochemical remission of acromegaly}

LV size and mass LV size (LVESD, LVEDD), wall thickness (IVST, PWT), and mass (LVM, LVMi) did not differ between the two groups (Table 2). LVH (defined as LVMi exceeding $49.2 \mathrm{~g} / \mathrm{m}^{2.7}$ for men and $46.7 \mathrm{~g} / \mathrm{m}^{2.7}$ for women (22)) was present in $50 \%$ of patients with GHD after acromegaly compared with $30 \%$ of patients with biochemical remission of acromegaly $(P=0.226)$. These results were confirmed after adjustment for age.

LV systolic function FS did not differ between the two groups. LVEF tended to be lower in patients with GHD after acromegaly compared with patients with biochemical remission $(P=0.070$, Fig. 1$)$. These results were not affected after adjustment for age $(P=0.030)$.

LV diastolic function No differences were noted in diastolic parameters ( $E$ and $A$ wave velocities, $E / A$ ratio) between the two groups. Additional data on diastolic function, as assessed by TDI, revealed that $E^{\prime}$ was lower in patients with GHD after acromegaly compared with patients with biochemical remission $(P=0.005$, Fig. 2$)$. Accordingly, $E^{\prime} / A^{\prime}$ ratio tended to be decreased $(P=0.079)$. These results were even more marked after adjustment for age $(P=0.001$ and $P=0.018$ for the $E^{\prime}$ and $E^{\prime} / A^{\prime}$ ratio respectively).

Heart valves Mitral regurgitation was absent in $81 \%$ of patients with $\mathrm{GHD}$, whereas $13 \%$ had trace, and $6 \%$ mild regurgitation, compared with 70,15 , and $15 \%$ respectively of patients with biochemical remission $(P=N S)$. Aortic regurgitation was absent in $88 \%$ of patients with GHD, whereas $13 \%$ had trace regurgitation, compared with 70 and $10 \%$ trace, and $20 \%$ mild regurgitation of patients with biochemical remission $(P=N S)$.

\section{GHD after acromegaly compared with healthy controls}

LV size and mass $L V$ size (LVESD, LVEDD), wall thickness (IVST, PWT), and mass (LVM, LVMi) did not differ between the two groups (Table 3). LVH (defined as LVMi exceeding $49.2 \mathrm{~g} / \mathrm{m}^{2.7}$ for men and $46.7 \mathrm{~g} / \mathrm{m}^{2.7}$ for women (22)) was present in $50 \%$ of patients with GHD after acromegaly compared with $38 \%$ of the healthy controls $(P=\mathrm{NS})$.

LV systolic function FS and LVEF did not differ between the two groups.

LV diastolic function $E$ wave velocity was lower in patients with GHD after acromegaly compared with healthy controls without any differences in $A$ wave

Table 2 Left ventricular dimensions, systolic function, and diastolic function in patients with GH deficiency (GHD) after acromegaly compared with patients with biochemical remission after acromegaly and patients with active acromegaly.

\begin{tabular}{|c|c|c|c|c|c|}
\hline & $\begin{array}{l}\text { GHD after acromegaly } \\
(n=16)\end{array}$ & $\begin{array}{l}\text { Biochemical remission } \\
\text { acromegaly }(n=20)\end{array}$ & $P$ value $^{\mathrm{a}}$ & $\begin{array}{l}\text { Active acromegaly } \\
(n=17)\end{array}$ & $\boldsymbol{P}$ value $^{\mathrm{a}}$ \\
\hline LVEDD (mm) & $51.6 \pm 6.1$ & $53.3 \pm 6.7$ & NS & $54.1 \pm 10.2$ & NS \\
\hline LVESD (mm) & $33.5 \pm 5.0$ & $34.2 \pm 6.6$ & NS & $37.5 \pm 10.5$ & NS \\
\hline IVST (mm) & $12.3 \pm 3.5$ & $10.2 \pm 2.4$ & NS & $13.5 \pm 3.9$ & NS \\
\hline PWT (mm) & $10.3 \pm 1.8$ & $9.7 \pm 1.7$ & NS & $10.7 \pm 2.3$ & NS \\
\hline FS (\%) & $36.8 \pm 5.8$ & $36.9 \pm 5.8$ & NS & $30.4 \pm 7.3$ & 0.014 \\
\hline LVEF (\%) & $65.9 \pm 7.3$ & $72.4 \pm 8.5$ & 0.070 & $58.8 \pm 9.3$ & 0.047 \\
\hline$E(\mathrm{~mm} / \mathrm{s})$ & $50.9 \pm 11.7$ & $56.0 \pm 15.0$ & NS & $56.0 \pm 16.2$ & NS \\
\hline$A(\mathrm{~mm} / \mathrm{s})$ & $59.6 \pm 17.7$ & $56.3 \pm 15.4$ & NS & $63.6 \pm 16.0$ & NS \\
\hline$E / A$ ratio & $0.9 \pm 0.2$ & $1.0 \pm 0.5$ & NS & $0.92 \pm 0.38$ & NS \\
\hline$E^{\prime}(\mathrm{cm} / \mathrm{s})$ & $6.0 \pm 2.1$ & $8.3 \pm 1.5$ & 0.005 & $6.0 \pm 2.4$ & NS \\
\hline$A^{\prime}(\mathrm{cm} / \mathrm{s})$ & $7.4 \pm 1.8$ & $7.8 \pm 1.8$ & NS & $8.1 \pm 2.9$ & NS \\
\hline$E^{\prime} / A^{\prime}$ ratio & $0.8 \pm 0.4$ & $1.1 \pm 0.4$ & 0.079 & $0.77 \pm 0.26$ & NS \\
\hline LVM (g) & $235.2 \pm 68.4$ & $211.8 \pm 84.5$ & NS & $289.8 \pm 158.3$ & NS \\
\hline LVMi $\left(\mathrm{g} / \mathrm{m}^{2.7}\right)$ & $50.9 \pm 15.3$ & $45.8 \pm 18.1$ & NS & $65.8 \pm 38.4$ & NS \\
\hline LVH (\%) & $50 \%$ & $30 \%$ & $N S^{b}$ & 71 & $\mathrm{NS}^{\mathrm{b}}$ \\
\hline
\end{tabular}

LVEDD, left ventricular end-diastolic diameter; LVESD, left ventricular end-systolic diameter; FS, fractional shortening; LVEF, left ventricular ejection fraction; $E, E$ wave (early filling phase); $A, A$ wave (atrial contraction); $E^{\prime}$, tissue Doppler $E$ wave; $A^{\prime}$, tissue Doppler $A$ wave; IVST, interventricular septum thickness; PWT, posterior wall thickness; LVMi, left ventricular mass index; LVH, left ventricular hypertrophy.

${ }^{a}$ ANOVA analysis with Tukey HSD correction for multiple comparisons was used to compare patients with GHD after acromegaly with patients with biochemical remission and patients with active acromegaly.

${ }^{b} \chi^{2}$ test. 


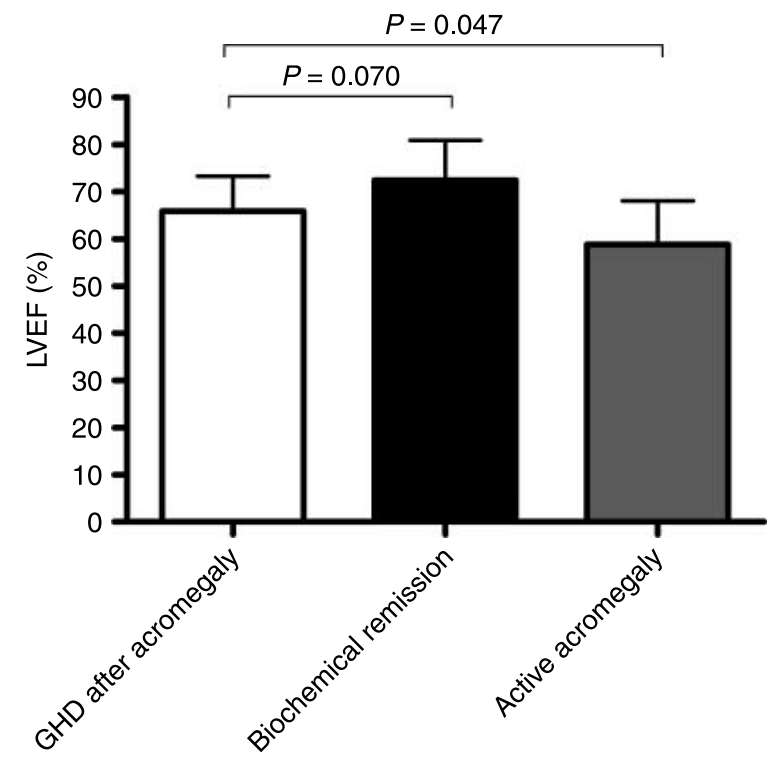

Figure 1 Systolic function, reflected by left ventricular ejection fraction (LVEF), was lower in patients with GHD acromegaly compared with patients with biochemical remission of acromegaly and increased compared with patients with active acromegaly.

velocity and $E / A$ ratio. Additional data on diastolic function, as assessed by TDI, revealed that $E^{\prime}$ was also lower in patients with GHD after acromegaly compared with healthy controls $(P=0.006$, Fig. 2). Accordingly, $E^{\prime} / A^{\prime}$ ratio tended to be decreased $(P=0.079)$.

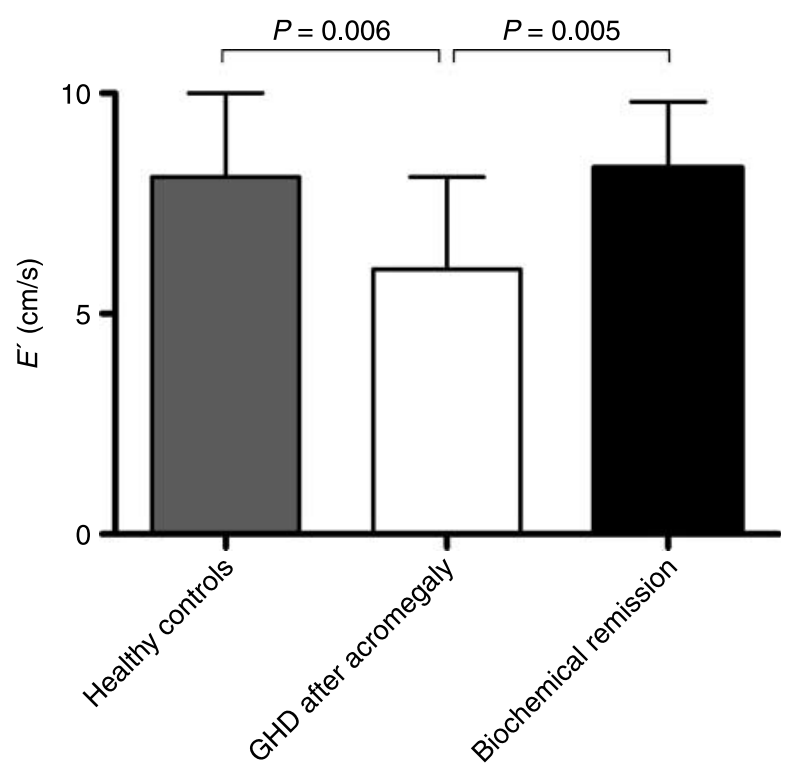

Figure $2 E^{\prime}$ as a marker of diastolic function (assessed by tissue Doppler imaging) was significantly lower in patients with $\mathrm{GH}$ deficiency (GHD) after acromegaly compared with healthy controls and patients with biochemical remission of acromegaly.
Table 3 Left ventricular dimensions, systolic function, and diastolic function in patients with GH deficiency (GHD) after acromegaly compared with healthy controls.

\begin{tabular}{llll}
\hline & $\begin{array}{l}\text { GHD after } \\
\text { acromegaly } \\
(n=16)\end{array}$ & $\begin{array}{l}\text { Healthy } \\
\text { controls } \\
(n=16)\end{array}$ & $\boldsymbol{P}^{\text {value }}$ \\
\hline LVEDD $(\mathrm{mm})$ & $51.6 \pm 6.1$ & $50.0 \pm 6.3$ & $\mathrm{NS}$ \\
LVESD $(\mathrm{mm})$ & $33.5 \pm 5.0$ & $30.6 \pm 4.4$ & $\mathrm{NS}$ \\
IVST $(\mathrm{mm})$ & $12.3 \pm 3.5$ & $10.8 \pm 1.9$ & $\mathrm{NS}$ \\
PWT $(\mathrm{mm})$ & $10.3 \pm 1.8$ & $10.6 \pm 1.5$ & $\mathrm{NS}$ \\
LVEF $(\%)$ & $65.9 \pm 7.3$ & $68.8 \pm 5.5$ & $\mathrm{NS}$ \\
FS $(\%)$ & $36.8 \pm 5.7$ & $38.8 \pm 4.4$ & $\mathrm{NS}$ \\
$E(\mathrm{~mm} / \mathrm{s})$ & $50.9 \pm 11.7$ & $62.5 \pm 17.4$ & 0.035 \\
$A(\mathrm{~mm} / \mathrm{s})$ & $59.6 \pm 17.7$ & $68.2 \pm 17.9$ & $\mathrm{NS}$ \\
$E / A \mathrm{ratio}$ & $0.90 \pm 0.24$ & $0.99 \pm 0.55$ & $\mathrm{NS}$ \\
$E^{\prime}(\mathrm{mm} / \mathrm{s})$ & $6.0 \pm 2.1$ & $8.1 \pm 1.9$ & 0.006 \\
$A^{\prime}(\mathrm{mm} / \mathrm{s})$ & $7.4 \pm 1.8$ & $7.8 \pm 2.4$ & $\mathrm{NS}$ \\
$E^{\prime} / A^{\prime} \mathrm{ratio}$ & $0.85 \pm 0.36$ & $1.27 \pm 0.85$ & 0.084 \\
LVM $(\mathrm{g})$ & $235.2 \pm 68.4$ & $207.9 \pm 55.4$ & $\mathrm{NS}$ \\
LVMi $\left(\mathrm{g} / \mathrm{m}^{2.7}\right)$ & $50.9 \pm 15.3$ & $44.3 \pm 9.8$ & $\mathrm{NS}$ \\
LVH $n(\%)$ & $8(50)$ & $6(38)$ & $\mathrm{NS}{ }^{\mathrm{b}}$ \\
\hline
\end{tabular}

LVEDD, left ventricular end-diastolic diameter; LVESD, left ventricular endsystolic diameter; FS, fractional shortening; LVEF, left ventricular ejection fraction; $E, E$ wave (early filling phase); $A, A$ wave (atrial contraction); $E^{\prime}$, tissue Doppler $E$ wave; $A^{\prime}$, tissue Doppler $A$ wave; IVST, interventricular septum thickness; PWT, posterior wall thickness; LVMi, left ventricular mass index; LVH, left ventricular hypertrophy. andependent samples $t$-test.

${ }^{\mathrm{b}} \chi^{2}$ test.

Heart valves Mitral regurgitation was absent in $81 \%$ of patients with GHD, whereas 13\% had trace, and $6 \%$ mild regurgitation, compared with 73,25 , and $6 \%$ respectively in healthy controls $(P=N S)$. Aortic regurgitation was absent in $88 \%$ of patients with GHD, whereas $13 \%$ had trace regurgitation, compared with $94 \%$ no and $13 \%$ trace regurgitation of healthy controls $(P=\mathrm{NS})$.

\section{GHD after acromegaly compared with active acromegaly}

LV size and mass LVESD and LVEDD did not differ between the two groups (Table 2, Fig. 1). Remarkably, IVST, PWT, and LVM were not different in patients with GHD after acromegaly compared with patients with active acromegaly. LVH (defined as LVMi exceeding $49.2 \mathrm{~g} / \mathrm{m}^{2.7}$ for men and $46.7 \mathrm{~g} / \mathrm{m}^{2.7}$ for women (22)) was present in $50 \%$ of patients with GHD after acromegaly compared with $71 \%$ of patients with active acromegaly $(P=N S)$. These results were confirmed after adjustment for age.

$\boldsymbol{L V}$ systolic function FS and LVEF were significantly higher in patients with GHD after acromegaly compared with patients with active acromegaly $(P=0.014$ and $P=0.047)$. These results were confirmed after adjustment for age $(P=0.005$ and $P=0.021$ for $\mathrm{FS}$ and LVEF respectively).

$\boldsymbol{L V}$ diastolic function No differences were observed in diastolic parameters ( $E$ and $A$ wave velocities, $E / A$ ratio) 
between the two groups. Diastolic function, assessed by TDI, did not reveal differences between the two groups. These results were confirmed after adjustment for age.

Heart valves The prevalence of mitral regurgitation was not different between the two groups (GHD: $81 \%$ absent, $13 \%$ trace, and $6 \%$ mild versus active acromegaly: $60 \%$ absent, $24 \%$ trace, $6 \%$ mild, and $12 \%$ severe (two patients), $P=\mathrm{NS}$ ). The prevalence of aortic regurgitation was also not different between the groups (GHD: $88 \%$ absent, $13 \%$ trace versus $71 \%$ absent, $6 \%$ trace, $18 \%$ mild, $6 \%$ severe, in active acromegaly, $P=\mathrm{NS}$ ).

\section{Multiple linear regression analysis}

Regression analysis was performed with systolic and diastolic function as dependent variables and age, BMI, IGF1 SDS, hypertension, and LVH as independent variables. All patients with acromegaly were included as one group in this analysis. Age was found to influence diastolic function as measured with conventional echocardiography $(\beta=0.386, P=0.021$ for $A$ and $\beta=-0.010, P=0.007$ for $E / A$ ratio). $\mathrm{LVH}$ and IGF1 SDS influenced diastolic function as measured with TDI $\left(\beta=-1.9, P=0.010\right.$ for LVH on $E^{\prime}$ and $\beta=$ $0.135, P=0.032$ for IGF1 SDS on $\left.A^{\prime}\right)$. We did not find any predictors for systolic function.

\section{Discussion}

In this study, we characterized cardiac function and morphology in patients with GHD after treatment for acromegaly. Because both acromegaly per se and GHD per se lead to specific structural and functional cardiac alterations, we wanted to assess to which extend GHD after previous exposure to $\mathrm{GH}$ excess influences cardiac parameters. This study indicates that GHD after acromegaly results in specific cardiac changes in diastolic function and that normal cardiac function is dependent on normal GH and IGF1 regulation.

To our knowledge, data on the cardiac manifestations of GHD after treatment for acromegaly have not been reported previously. In active acromegaly, a specific cardiomyopathy develops characterized by concentric $\mathrm{LVH}$, and LV systolic and diastolic dysfunction. Adequate treatment with stringent control of $\mathrm{GH}$ and IGF1 levels ameliorates signs and symptoms of acromegalic cardiomyopathy. Successful transsphenoidal surgery tends to reverse LVH and to improve diastolic function (25). A recent meta-analysis, which evaluated the impact of this treatment on the heart in acromegaly, demonstrated that somatostatin analog treatment (with a duration ranging from a few days to 18 months) consistently improved markers of LVH (LVM index, IVST, and LV PWT) and diastolic function (26). The findings in our patients with active acromegaly compared with those with biochemical remission are in line with the data from these intervention studies.

Treatment of acromegaly, however, can result in GHD in some patients, especially after previous radiotherapy (5). Several parameters of cardiac morphology and function were altered in our patients with GHD after acromegaly.

First, systolic function at rest tended to be decreased compared with patients with biochemical remission. However, when compared with healthy controls systolic function was not affected in GHD after acromegaly. Therefore, we should be careful in interpreting this trend, since previous acromegaly might have influenced systolic function in patients with biochemical remission. Systolic function was found to be decreased in patients with adult-onset GHD not previously exposed to GH excess. This was found to be correlated with both age and the severity of GHD $(7,8)$. In addition, we noted that systolic function was lower in patients with active acromegaly than in those with GHD after acromegaly. Hypertension and LVH are major determinants of systolic function. About $41 \%$ of patients with active acromegaly suffered from hypertension and $71 \%$ had LVH compared with 38 and 50\% in patients with GHD respectively. Apparently, most probably among many others, these factors result in cardiac systolic function being more affected in states of GH excess than in GHD.

Secondly, TDI revealed a decrease in parameters reflecting diastolic function in patients with GHD after acromegaly compared with those with biochemical remission and healthy controls. To our knowledge, only one study assessed diastolic function in adults with GHD with TDI (13). In that study, $E^{\prime}$ was decreased compared with controls (13), in line with the observed decrease in $E^{\prime}$ in our patients. In active acromegaly, however, diastolic function was also affected (15). Indeed, there was no difference in diastolic function in patients with GHD after acromegaly compared with patients with active acromegaly.

Indices of LVM, wall thickness, and LV diameters were unaltered in patients with GHD after acromegaly compared with patients with biochemical remission of acromegaly and healthy controls. Indeed, in patients with adult-onset GHD due to other diseases, IVST does not differ from healthy controls (7). However, in adults with childhood-onset GHD, it was found to be decreased $(9,10)$. LVM was unaffected in our patients with GHD after acromegaly compared with patients with biochemical remission of acromegaly and compared with patients with active acromegaly. Several studies in patients with childhood-onset GHD revealed a decreased LVM $(6,9,10)$, whereas it was unaffected in patients with adult-onset GHD, as was the case in our patients (7).

The effects of rhGH on the myocardium in adults with GHD without previous exposure to acromegaly have been reported in a meta-analysis (27). The rhGH replacement with a maximum duration of 18 months 
increased LVM and IVST, whereas diastolic function was not affected (27). Additionally, a trend in improvement in FS was observed (27). It is unknown, however, whether these beneficial changes can also occur in patients with GHD induced by previous treatment for active acromegaly.

In conclusion, GHD after acromegaly results in specific cardiac alterations in diastolic function. In addition, systolic function tended to be decreased in patients with GHD after acromegaly compared with patients with biochemical remission but not when compared with healthy controls, but was higher than in patients with active acromegaly. This study shows that normal cardiac function is dependent on normal GH and IGF1 regulation. It remains to be determined whether these specific cardiac changes after previous prolonged exposure to $\mathrm{GH}$ excess followed by GHD affect the response to rhGH replacement.

\section{Declaration of interest}

There is no conflict of interest that could be perceived as prejudicing the impartiality of the research reported.

\section{Funding}

A A van der Klaauw is supported by an AGIKO grant of the Netherlands Organisation for Health Research and Development (grant number: 92003423). The other authors have nothing to disclose.

\section{References}

1 Colao A, Ferone D, Marzullo P \& Lombardi G. Systemic complications of acromegaly: epidemiology, pathogenesis, and management. Endocrine Reviews 200425 102-152.

2 Biermasz NR, Dekker FW, Pereira AM, van Thiel SW, Schutte PJ, van Dulken H, Romijn JA \& Roelfsema F. Determinants of survival in treated acromegaly in a single center: predictive value of serial insulin-like growth factor I measurements. Journal of Clinical Endocrinology and Metabolism 200489 2789-2796.

3 Colao A, Marzullo P, Ferone D, Spinelli L, Cuocolo A, Bonaduce D, Salvatore M, Boerlin V, Lancranjan I \& Lombardi G. Cardiovascular effects of depot long-acting somatostatin analog Sandostatin LAR in acromegaly. Journal of Clinical Endocrinology and Metabolism $2000853132-3140$.

4 Colao A, Cuocolo A, Marzullo P, Nicolai E, Ferone D, Della Morte AM, Pivonello R, Salvatore M \& Lombardi G. Is the acromegalic cardiomyopathy reversible? Effect of 5-year normalization of growth hormone and insulin-like growth factor I levels on cardiac performance Journal of Clinical Endocrinology and Metabolism 200186 1551-1557.

5 van der Klaauw AA, Pereira AM, van Thiel SW, Smit JW, Corssmit EP, Biermasz NR, Frolich M, Iranmanesh A, Veldhuis JD, Roelfsema F \& Romijn JA. GH deficiency in patients irradiated for acromegaly: significance of GH stimulatory tests in relation to the $24 \mathrm{~h}$ GH secretion. European Journal of Endocrinology $2006154851-858$.

6 Amato G, Carella C, Fazio S, La Montagna G, Cittadini A, Sabatini D, Marciano-Mone C, Sacca L \& Bellastella A. Body composition, bone metabolism, and heart structure and function in growth hormone $(\mathrm{GH})$-deficient adults before and after $\mathrm{GH}$ replacement therapy at low doses. Journal of Clinical Endocrinology and Metabolism 199377 1671-1676.
7 Colao A, Cuocolo A, Di Somma C, Cerbone G, Della Morte AM, Nicolai E, Lucci R, Salvatore M \& Lombardi G. Impaired cardiac performance in elderly patients with growth hormone deficiency. Journal of Clinical Endocrinology and Metabolism 1999 84 3950-3955.

8 Colao A, Di Somma C, Cuocolo A, Filippella M, Rota F, Acampa W, Savastano S, Salvatore M \& Lombardi G. The severity of growth hormone deficiency correlates with the severity of cardiac impairment in 100 adult patients with hypopituitarism: an observational, case-control study. Journal of Clinical Endocrinology and Metabolism 200489 5998-6004.

9 Merola B, Cittadini A, Colao A, Longobardi S, Fazio S, Sabatini D, Sacca L \& Lombardi G. Cardiac structural and functional abnormalities in adult patients with growth hormone deficiency. Journal of Clinical Endocrinology and Metabolism $1993771658-1661$.

10 Sartorio A, Ferrero S, Conti A, Bragato R, Malfatto G, Leonetti G \& Faglia G. Adults with childhood-onset growth hormone deficiency: effects of growth hormone treatment on cardiac structure. Journal of Internal Medicine 1997 241 515-520.

11 Colao A, Cuocolo A, Di Somma C, Cerbone G, Morte AM, Pivonello R, Nicolai E, Salvatore M \& Lombardi G. Does the age of onset of growth hormone deficiency affect cardiac performance? A radionuclide angiography study Clinical Endocrinology $2000 \mathbf{5 2} 447-455$.

12 Erdogan D, Tukek T, Aral F, Oflaz H, Ozaydin M, Kocaman O, Akkaya V, Goren T \& Molvalilar S. Structural, functional and autonomic changes in the cardiovascular system in growth hormone deficient patients. Annals of Noninvasive Electrocardiology 20049 19-23.

13 Yurci A, Oflaz H, Meric M \& Ozbey N. Mitral and tricuspid annular velocities determined by Doppler tissue imaging in hypopituitary, growth hormone-deficient patients. Hormone Research $2005 \mathbf{6 4}$ 107-114.

14 Pereira AM, van Thiel SW, Lindner JR, Roelfsema F, van der Wall EE, Morreau H, Smit JW, Romijn JA \& Bax JJ. Increased prevalence of regurgitant valvular heart disease in acromegaly. Journal of Clinical Endocrinology and Metabolism 2004 89 71-75.

15 van Thiel SW, Bax JJ, Biermasz NR, Holman ER, Poldermans D, Roelfsema F, Lamb HJ, van der Wall EE, Smit JW, Romijn JA \& Pereira AM. Persistent diastolic dysfunction despite successful long-term octreotide treatment in acromegaly. European Journal of Endocrinology $2005153231-238$.

16 Growth Hormone Research Society. Consensus guidelines for the diagnosis and treatment of adults with growth hormone deficiency: summary statement of the Growth Hormone Research Society Workshop on Adult Growth Hormone Deficiency. Journal of Clinical Endocrinology and Metabolism $1998 \mathbf{8 3}$ 379-381.

17 Zanettini R, Antonini A, Gatto G, Gentile R, Tesei S \& Pezzoli G. Valvular heart disease and the use of dopamine agonists for Parkinson's disease. New England Journal of Medicine 2007356 39-46.

18 Schiller NB, Shah PM, Crawford M, DeMaria A, Devereux R, Feigenbaum H, Gutgesell H, Reichek N, Sahn D \& Schnittger I. Recommendations for quantitation of the left ventricle by twodimensional echocardiography. American Society of Echocardiography Committee on Standards, Subcommittee on Quantitation of Two-Dimensional Echocardiograms. Journal of the American Society of Echocardiography 19892 358-367.

19 Perry GJ, Helmcke F, Nanda NC, Byard C \& Soto B. Evaluation of aortic insufficiency by Doppler color flow mapping. Journal of the American College of Cardiology 19879 952-959.

20 Thomas JD. How leaky is that mitral valve? Simplified Doppler methods to measure regurgitant orifice area Circulation 199795 548-550.

21 Devereux RB, Alonso DR, Lutas EM, Gottlieb GJ, Campo E, Sachs I \& Reichek N. Echocardiographic assessment of left ventricular hypertrophy: comparison to necropsy findings. American Journal of Cardiology $1986 \mathbf{5 7}$ 450-458.

22 Vitale G, Galderisi M, Pivonello R, Spinelli L, Ciccarelli A, de Divitiis O, Lombardi G \& Colao A. Prevalence and determinants of left ventricular hypertrophy in acromegaly: impact of different methods of indexing left ventricular mass. Clinical Endocrinology $200460343-349$. 
23 Cole TJ. The LMS method for constructing normalized growth standards. European Journal of Clinical Nutrition $1990 \mathbf{4 4}$ 45-60.

24 Rikken B, van Doorn J, Ringeling A, Van den Brande JL, Massa G \& Wit JM. Plasma levels of insulin-like growth factor (IGF)-I, IGF-II and IGF-binding protein-3 in the evaluation of childhood growth hormone deficiency. Hormone Research 199850 166-176.

25 Jaffrain-Rea ML, Minniti G, Moroni C, Esposito V, Ferretti E, Santoro A, Infusino T, Tamburrano G, Cantore G \& Cassone R. Impact of successful transsphenoidal surgery on cardiovascular risk factors in acromegaly. European Journal of Endocrinology 2003 148 193-201.
26 Maison P, Tropeano AI, Macquin-Mavier I, Giustina A \& Chanson P. Impact of somatostatin analogs on the heart in acromegaly: a metaanalysis. Journal of Clinical Endocrinology and Metabolism 200792 1743-1747.

27 Maison P \& Chanson P. Cardiac effects of growth hormone in adults with growth hormone deficiency: a meta-analysis. Circulation 2003108 2648-2652.

Received 27 August 2008

Accepted 31 August 2008 